\title{
Induced volatiles in the interaction between soybean (Glycine max) and the Mexican soybean weevil (Rhyssomatus nigerrimus)
}

\author{
K. Espadas-Pinacho ${ }^{a}$, G. López-Guillén ${ }^{b}$ (D), J. Gómez-Ruiz ${ }^{a}$ (D) and L. Cruz-López ${ }^{a *}$ (D) \\ ${ }^{a}$ El Colegio de la Frontera Sur - ECOSUR, Grupo de Ecología de Artrópodos y Manejo de Plagas, Tapachula, \\ Chiapas, Mexico \\ bInstituto Nacional de Investigaciones Forestales, Agrícolas y Pecuarias - INIFAP, Campo Experimental Rosario Izapa, \\ Tuxtla Chico, Chiapas, Mexico \\ *e-mail: 1cruz@ecosur.mx
}

Received: August 10, 2019 - Accepted: February 26, 2020 - Distributed: August 31, 2021

(With 5 figures)

\begin{abstract}
The present study analyzed the volatile compounds emitted by Glycine max (cv. FT-Cristalina-RCH) soybean plants: healthy plants and plants damaged mechanically or by the Mexican soybean weevil Rhyssomatus nigerrimus. The SPME method was used to compare the volatile profile of soybean plants in four different conditions. The volatile profile of G. max plants infested by R. nigerrimus was qualitatively and quantitatively different from that of healthy and mechanically damaged plants. Emission of 59 compounds was detected in the four treatments. Of these compounds, 19 were identified by comparison of the Kovats index, mass spectrum and retention times with those of synthetic standards. An increase in concentration of the volatiles (Z)-3-hexenyl acetate and the compound 1-octen-3-ol was observed when the soybean plants were mechanically damaged. The compounds mostly produced by the soybean plant during infestation by male and female $R$. nigerrimus were 1-octen-3-ol, 6-methyl-5-hepten-2-one, $(E)$ - $\beta$-ocimene, salicylaldehyde, unknown 10, linalool, methyl salicylate, $(Z)-8$-dodecenyl acetate (ester 5), ketone 2 and geranyl acetone. Behavioral effects of the identified compounds during the insect-plant interaction and their conspecifics are discussed.
\end{abstract}

Keywords: SPME, limonene, methyl salicylate, linalool, (E)- $\beta$-ocimene.

\section{Voláteis induzidos na interação entre soja (Glycine max) e gorgulho da soja mexicana (Rhyssomatus nigerrimus)}

\section{Resumo}

O presente estudo analisou os compostos voláteis emitidos pelas plantas de soja Glycine max (cv. FT-Cristalina-RCH): plantas e plantas sadias danificadas mecanicamente ou pelo gorgulho da soja mexicana Rhyssomatus nigerrimus. O método SPME foi utilizado para comparar o perfil volátil de plantas de soja em quatro diferentes condições. O perfil volátil das plantas de G. max infestadas por $R$. nigerrimus foi qualitativa e quantitativamente diferente do das plantas saudáveis e danificadas mecanicamente. Foi detectada, nos quatro tratamentos, emissão de 59 compostos, dos quais 19 foram identificados por comparação do índice de Kovats, espectro de massa e tempos de retenção com aqueles de padrões sintéticos. Um aumento na concentração dos voláteis acetato de (Z)-3-hexil e do composto 1-octeno-3-ol foi observado quando as plantas de soja foram mecanicamente danificadas. Os compostos produzidos principalmente pela planta de soja durante a infestação por $R$. nigerrimus macho e fêmea foram 1-octeno-3-ol, 6-metil-5-hepteno-2-ona, (E)- $\beta$-ocimeno, salicilaldeído, desconhecido 10, linalol, salicilato de metila, acetato de (Z)-8-dodecenila (éster 5), cetona 2 e geranil acetona. Foram discutidos os efeitos comportamentais dos compostos identificados durante a interação inseto-planta e seus coespecíficos.

Palavras-chave: SPME, limoneno, salicilato de metila, linalol, (E)- $\beta$-ocimeno.

\section{Introduction}

Plants produce and emit volatile compounds (VC) that are released by flowers, fruits and vegetative tissues (Marín and Céspedes, 2007). Generally, these substances are made of terpenes derived from fatty acids and aromatic compounds. Plant volatiles act as signals for other organisms and for the plant itself. They can also be exported and modify the surroundings of the plant species that produce them, its neighbors and its enemies (Rostás and Eggert, 2008). The main functions of VC in plants are to attract pollinators, seed dispersers, and natural enemies of herbivores; to be 
intraspecific and interspecific messengers; and to defend the plants by repelling insects or detaining colonization of pathogenic bacteria and fungi (Byers et al., 2014). VC can also be used by herbivores as cues to direct their flight to host plants (Visser, 1986).

$\mathrm{VC}$ are classified into two types according to the way in which they are released: constitutive and induced. Constitutive VC are those inherent to the plant, while induced $\mathrm{VC}$ are emitted by plants in response to a stimulus caused by mechanical damage or damage by herbivore insects to plant tissues (Sánchez and Délano, 2003). When the latter type of damage occurs, the emitted compounds are called herbivore-induced plant volatiles (HIPV) (Vivanco et al., 2005; Rostás and Eggert, 2008).

The volatiles induced by herbivores are emitted at the damaged site as well as in undamaged tissues (War et al. 2011). The plant uses these metabolites as chemical defense; outstanding of these metabolites are toxins, repellents, anti-feeding compounds and digestibility reducers (Moraes et al., 2000). Recent studies indicate that compounds released by a plant vary with species, plant variety and herbivore species (Michereff et al., 2011).

This study focuses on volatiles induced by herbivores that attack soybean Glycine max L. (Fabales: Fabaceae), which is cultivated worldwide and is economically important for production of oil and biodiesel (FAO, 2017). In the state of Chiapas, Mexico, soybean cultivation has extended over an area of 130 ha with a production of $273 \mathrm{t}$ of soybeans and yield of $2.11 \mathrm{t} / \mathrm{ha}$ (SIAP, 2017).

Volatile compounds reported in soybean have been in seeds which are 3-hexanone, (E)-2-hexanal, 1-hexanol, 3-octanone, hexanal, $(E)$-2-heptenal, $(E)$-2-octenal, ethanol, 1-hexanol and 1-octen-3-ol (Boué et al., 2003). When attacked by Spodoptera frugiperda (J. E. Smith) (Lepidoptera: Noctuidae), Euschistus heros (Fabricius) (Hemiptera: Pentatomidae) and Aphis glycines Matsumura (Hemiptera: Aphididae), the plants release induced systemic volatile compounds (Moraes et al., 2005; Zhu and Park, 2005; Rostás and Eggert, 2008; Cortés, 2016).

In the Mexican state of Tamaulipas, San Luis Potosí, Veracruz and Chiapas, the major economically important pest of soybeans is the Mexican soybean weevil Rhyssomatus nigerrimus Fahraeus 1837 (Coleoptera: Curculionidae) (Terán-Vargas and López-Guillén, 2014). Cortés (2016) reported that this insect is attracted by volatiles emitted by the soybean plant. However, differences in the VC profile have been observed between healthy plants and plants attacked by the weevil (Cruz-López, unpublished data).

Although the volatile profile of soybean plants damaged by other pests is known, there are no reports regarding the curculionid $R$. nigerrimus. Therefore, the objective of this study was to identify the volatile compounds produced in leaves and pods of the soybean plant when it is attacked by $R$. nigerrimus, an insect considered a key pest of soybean crops in the Soconusco region, Chiapas. The knowledge generated in this study will provide useful information for future biological tests on attraction between plant and insect, which will eventually favor development of attractants for control and monitoring of the soybean weevil.

\section{Material and Methods}

\subsection{Biological material}

$R$. nigerrimus adults were collected manually from soybean crops in the Ejido el Manzano in the municipality of Tapachula, Chiapas, every week for four months. These specimens were stored in plastic recipients covered with organdy fabric for later transfer to the chemical ecology insectary of El Colegio de la Frontera Sur (ECOSUR). In the laboratory, the collected insects were separated by sex using a stereoscopic microscope following the method proposed by López-Guillén et al. (2016). Female and male $R$. nigerrimus were kept separately in plastic containers and fed sweet potato slices (Ipomoea batatas L.). The insects were kept in the ECOSUR insectary at a temperature of $25 \pm 2{ }^{\circ} \mathrm{C}$ and relative humidity (RH) of $70 \pm 5 \%$ with a $12: 12 \mathrm{~h}$ light:dark photoperiod.

The soybean plants (cv. FT-Cristalina-RCH) used in the experiments were planted in pots containing Miracle-Gro ${ }^{\circledR}$ substrate and watered daily. The experiment was conducted in a room with controlled climate $\left(25 \pm 2{ }^{\circ} \mathrm{C}, 60 \pm 10 \% \mathrm{RH}\right)$. The pods used in the experiments were obtained from the soybean plants grown to the R6 state.

\subsection{Sampling of volatiles in the laboratory}

Soybean plant emitted compounds were collected by solid phase microextraction (SPME). Four treatments were set up. The first treatment consisted of introducing 30 adult $R$. nigerrimus females or males into a $125 \mathrm{~mL}$ Erlenmeyer flask with a soybean plant. In the second treatment 30 adult $R$. nigerrimus males were introduced into the flask without soybean plant. The third treatment a flask held a soybean plant with mechanical damage in the trifolio with a $1 \mathrm{~mm}$ diameter, $250 \mathrm{~mm}$ long entomological needle. The fourth treatment a control flask held a healthy soybean plant. Soybean pods emitted compounds were collected by SPME. Four treatments were set up. In the first treatment 30 adult females or males of $R$. nigerrimus were added into a $10 \mathrm{~mL}$ vial containing 12 grams of soybean pods, in the second treatment 30 adult males of $R$. nigerrimus were placed in the vial without pods, the third treatment consisted of a vial containing 12 grams of pods mechanically damaged with an entomological needle of $1 \mathrm{~mm}$ in diameter and a length of $250 \mathrm{~mm}$, and the fourth treatment was a vial containing 12 grams of healthy soybean pods used as a control. Six repetitions were performed for each treatment.

The entrance of the flask or vial was covered with aluminum foil and the SPME device containing the $65 \mu \mathrm{m}$ polydimethylsiloxane-divinylbenzene (PDMS/DVB) (Supelco ${ }^{\circledR}$, Toluca, Mexico) fiber was introduced. The fiber had been previously heated to $250^{\circ} \mathrm{C}$ for $5 \mathrm{~min}$ in the gas chromatograph (GC) to prevent possible contamination by volatiles from the previous sample absorbed in the fiber. The PDMS/DVB fiber was left for $24 \mathrm{~h}$ to absorb the 
volatiles, after which time it was removed. The volatile capture room was at $25 \pm 2{ }^{\circ} \mathrm{C}$ and 50 to $60 \%$ RH. Illumination was provided by four $39 \mathrm{~W}$ fluorescent lamps located $3 \mathrm{~m}$ above the volatile collection devices.

\subsection{Chemical analysis}

Desorption of the captured volatile compounds were desorbed inside the injection port of the gas chromatograph for $1 \mathrm{~min}$ at $250^{\circ} \mathrm{C}$. The volatile compounds captured by SPME were identified chemically in a gas chromatograph (Varian CP/3800) coupled to a mass spectrometer (Varian Saturn 2200), using a cast non-polar SPB-1 capillary column $30 \mathrm{~m}$ long, $0.25 \mathrm{~mm}$ interior diameter (Supelco ${ }^{\circledR}$, Toluca, Mexico). Analysis was performed with a temperature ramp beginning with an initial temperature of $50^{\circ} \mathrm{C}$ (for $2 \mathrm{~min}$ ), increasing $15^{\circ} \mathrm{C}$ every min until reaching $280^{\circ} \mathrm{C}$ held for $10 \mathrm{~min}$. Helium was the carrier gas and injector temperature was $250^{\circ} \mathrm{C}$. Ionization was carried out by electron impact at $70 \mathrm{eV}$. The compounds were identified by comparing the retention index, mass spectra and retention times with those of synthetic standards. Other compounds were tentatively identified based on comparison with spectra from the National Institute of Standards and Technology (NIST) library, version 2.0.

\subsection{Statistical analysis}

Data were analyzed using the classification type random forest multivariate technique (Liaw \& Wiener, 2002) with R software (R Development Core Team) to determine associations among the compositions of induced volatiles and to compare the treatments applied to soybean plants and pods.

\section{Results}

SPME analysis of the samples by gas chromatography-mass spectrometry shows that the volatile compounds emitted by soybean plant and pods include aromatics, esters, terpenes, ketones, alcohols and aldehydes (Tables 1 and 2).

A total of 59 compounds produced in the four treatments were detected. Of these compounds, we were able to identify 19 volatile compounds by comparison of the Kovats index, mass spectra and retention times with those of synthetic standards. Figure 1 shows the structures of the identified compounds.

Table 1. Volatile compounds identified in the treatments applied to soybean plants, cv. FT-Cristalina-RCH (proportions \pm standard error).

\begin{tabular}{|c|c|c|c|c|c|c|}
\hline Num. & Compound & IR & $\begin{array}{c}\text { Plant with } \\
\text { males }\end{array}$ & $\begin{array}{c}\text { Plant with } \\
\text { females }\end{array}$ & $\begin{array}{l}\text { Plant with } \\
\text { mechanical } \\
\text { damage }\end{array}$ & $\begin{array}{l}\text { Healthy } \\
\text { plant }\end{array}$ \\
\hline 1 & $\alpha$-Pinene* & 919.84 & $0.45 \pm 0.13$ & ND & $1.41 \pm 0.27$ & $0.20 \pm 0.05$ \\
\hline 2 & Pseudocumene $(1,2,4$-TMB)* & 943.53 & $0.37 \pm 0.26$ & ND & $0.30 \pm 0.06$ & $0.07 \pm 0.03$ \\
\hline 3 & Derivate of benzene 1 & 951.95 & $0.67 \pm 0.27$ & $0.36 \pm 0.09$ & $1.40 \pm 0.15$ & $0.43 \pm 0.08$ \\
\hline 4 & Derivate of benzene 2 & 955.45 & $0.31 \pm 0.12$ & $0.15 \pm 0.07$ & $1.40 \pm 0.35$ & $0.11 \pm 0.04$ \\
\hline 5 & 1-Octen-3-ol* & 971.63 & $9.80 \pm 2.68$ & $8.25 \pm 1.66$ & $15.34 \pm 1.84$ & $3.14 \pm 1.41$ \\
\hline 6 & 6-Methyl-5-hepten-2-one* & 976.54 & $4.90 \pm 0.84$ & $2.51 \pm 0.60$ & $4.24 \pm 0.37$ & $2.69 \pm 0.91$ \\
\hline 7 & 2-Octanone* & 981.71 & $1.56 \pm 0.37$ & $0.86 \pm 0.39$ & ND & ND \\
\hline 8 & Mesitylene* & 988.91 & $2.06 \pm 0.40$ & $1.59 \pm 0.32$ & $4.25 \pm 0.42$ & $1.41 \pm 0.18$ \\
\hline 9 & (Z)-3-Hexenyl acetate* & 996.28 & $2.28 \pm 0.53$ & $0.81 \pm 0.53$ & $14.22 \pm 3.78$ & $1.64 \pm 0.18$ \\
\hline 10 & Terpene 1 & $1,003.74$ & $0.20 \pm 0.07$ & $0.07 \pm 0.04$ & $0.62 \pm 0.12$ & $0.07 \pm 0.03$ \\
\hline 11 & Cymene isomer 1 & $1,015.23$ & $0.37 \pm 0.18$ & $0.23 \pm 0.08$ & $0.51 \pm 0.08$ & $0.36 \pm 0.04$ \\
\hline 12 & Hemellitol $(1,2,3-\mathrm{TMB})^{*}$ & $1,019.79$ & $0.22 \pm 0.06$ & $0.20 \pm 0.07$ & $0.71 \pm 0.10$ & $0.29 \pm 0.06$ \\
\hline 13 & Ethyl hexanol* & $1,024.21$ & $0.45 \pm 0.10$ & $0.64 \pm 0.17$ & $0.87 \pm 0.15$ & $0.55 \pm 0.30$ \\
\hline 14 & (Z)- $\beta$-Ocimene* & $1,026.69$ & $1.89 \pm 1.33$ & $2.90 \pm 2.54$ & $1.14 \pm 0.20$ & $1.07 \pm 0.57$ \\
\hline 15 & Limonene* & $1,030.02$ & $0.58 \pm 0.19$ & $0.93 \pm 0.35$ & ND & $0.40 \pm 0.19$ \\
\hline 16 & Unknown 1 & $1,036.49$ & ND & ND & $0.42 \pm 0.13$ & $0.07 \pm 0.02$ \\
\hline 17 & $(E)-\beta$-Ocimene* & $1,042.08$ & $3.26 \pm 1.86$ & $10.19 \pm 4.52$ & $0.29 \pm 0.11$ & $6.27 \pm 5.0$ \\
\hline 18 & Salicylaldehyde** & $1,049.96$ & $5.61 \pm 2.78$ & $1.64 \pm 0.50$ & ND & ND \\
\hline 19 & Cymene isomer 2 & $1,056.54$ & $0.99 \pm 0.27$ & $0.49 \pm 0.10$ & $0.64 \pm 0.20$ & $0.78 \pm 0.15$ \\
\hline 20 & Unknown 2 & $1,066.95$ & ND & ND & $3.23 \pm 0.61$ & $0.42 \pm 0.11$ \\
\hline 21 & Cymene isomer 3 & $1,076.57$ & $0.42 \pm 0.13$ & $0.62 \pm 0.33$ & $0.59 \pm 0.05$ & $0.70 \pm 0.36$ \\
\hline 22 & Cymene isomer 4 & $1,080.01$ & $0.43 \pm 0.13$ & $0.38 \pm 0.14$ & $0.56 \pm 0.04$ & $0.28 \pm 0.09$ \\
\hline 23 & Alkene 1 & $1,087.51$ & $2.83 \pm 1.11$ & $4.48 \pm 1.96$ & ND & ND \\
\hline 24 & Hydrocarbon 1 & $1,095.01$ & $0.97 \pm 0.39$ & $0.83 \pm 0.48$ & $0.83 \pm 0.08$ & $0.80 \pm 0.16$ \\
\hline 25 & Linalool* & $1,098.89$ & $1.00 \pm 0.29$ & $5.267 \pm 2.17$ & $1.68 \pm 0.39$ & ND \\
\hline 26 & 2-Nonen-1-ol & $1,104.24$ & $4.53 \pm 1.72$ & $3.26 \pm 0.82$ & $6.14 \pm 0.29$ & $7.65 \pm 1.53$ \\
\hline
\end{tabular}

$\mathrm{IR}=$ Retention index; $\mathrm{ND}=$ Not Detected; $*=$ Confirmed with synthetics; $* *=$ Compared with the NIST library; TMB=Trimethylbenzene. 
Table 1. Continued...

\begin{tabular}{|c|c|c|c|c|c|c|}
\hline Num. & Compound & IR & $\begin{array}{c}\text { Plant with } \\
\text { males }\end{array}$ & $\begin{array}{l}\text { Plant with } \\
\text { females }\end{array}$ & $\begin{array}{c}\text { Plant with } \\
\text { mechanical } \\
\text { damage }\end{array}$ & $\begin{array}{l}\text { Healthy } \\
\text { plant }\end{array}$ \\
\hline 27 & Unknown 3 & $1,128.99$ & ND & $0.16 \pm 0.06$ & ND & ND \\
\hline 28 & Carveol & $1,158.33$ & $0.45 \pm 0.08$ & $0.85 \pm 0.20$ & $0.97 \pm 0.42$ & $0.72 \pm 0.13$ \\
\hline 29 & Aldehyde 1 & $1,172.22$ & $1.75 \pm 0.62$ & $1.93 \pm 0.34$ & $3.06 \pm 0.21$ & $3.83 \pm 0.57$ \\
\hline 30 & Ketone 1 & $1,192.98$ & $0.44 \pm 0.24$ & $0.70 \pm 0.39$ & ND & ND \\
\hline 31 & Hydrocarbon 2 & $1,197.27$ & $0.82 \pm 0.45$ & $0.84 \pm 0.35$ & $1.05 \pm 0.17$ & $0.64 \pm 0.07$ \\
\hline 32 & Unknown 4 & $1,201.71$ & ND & ND & $0.35 \pm 0.23$ & $0.73 \pm 0.17$ \\
\hline 33 & Methyl salicylate* & $1,204.43$ & $1.96 \pm 1.00$ & $3.35 \pm 1.40$ & ND & ND \\
\hline 34 & Aldehyde 2 & $1,207.75$ & $9.43 \pm 4.05$ & $7.32 \pm 1.53$ & $14.23 \pm 1.53$ & $28.90 \pm 6.01$ \\
\hline 35 & Derivate of cyclohexanone & $1,269.72$ & $0.44 \pm 0.10$ & $0.98 \pm 0.17$ & $1.20 \pm 0.25$ & ND \\
\hline 36 & Geranial* & $1,276.18$ & $0.34 \pm 0.13$ & $1.29 \pm 065$ & $0.74 \pm 0.08$ & $0.65 \pm 0.33$ \\
\hline 37 & Ester 1 & $1,280.89$ & $0.58 \pm 0.29$ & ND & ND & ND \\
\hline 38 & Unknown 5 & $1,282.83$ & ND & $0.39 \pm 0.13$ & $0.76 \pm 0.17$ & $1.48 \pm 0.28$ \\
\hline 39 & 2-Undecanone* & $1,296.23$ & $0.24 \pm 0.08$ & $0.49 \pm 0.17$ & ND & ND \\
\hline 40 & Hydrocarbon 3 & $1,299.09$ & $2.02 \pm 1.09$ & ND & ND & ND \\
\hline 41 & Unknown 6 & $1,299.98$ & ND & $0.84 \pm 0.16$ & $0.98 \pm 0.11$ & $0.88 \pm 0.16$ \\
\hline 42 & Hydrocarbon 4 & $1,308.28$ & $0.09 \pm 0.04$ & $0.21 \pm 0.13$ & $0.33 \pm 0.16$ & $0.08 \pm 0.04$ \\
\hline 43 & Aldehyde 3 & $1,312.33$ & $0.67 \pm 0.33$ & $0.21 \pm 0.10$ & $0.99 \pm 0.11$ & $2.22 \pm 0.32$ \\
\hline 44 & Unknown 7 & $1,315.23$ & ND & $0.97 \pm 0.77$ & ND & ND \\
\hline 45 & Ester 2 & $1,325.45$ & $0.34 \pm 0.18$ & $0.73 \pm 0.32$ & ND & ND \\
\hline 46 & Ester 3 & $1,361.66$ & $2.10 \pm 0.20$ & $2.34 \pm 0.97$ & $1.37 \pm 0.17$ & $4.24 \pm 0.76$ \\
\hline 47 & Unknown 8 & $1,377.85$ & ND & ND & $0.88 \pm 0.26$ & $1.16 \pm 0.39$ \\
\hline 48 & Ester 4 & $1,383.2$ & $2.24 \pm 0.14$ & $0.96 \pm 0.45$ & $2.06 \pm 0.29$ & $4.17 \pm 0.89$ \\
\hline 49 & (Z)-8-Dodecenyl acetate (Ester 5) & $1,386.47$ & $10.77 \pm 3.96$ & $11.42 \pm 6.10$ & ND & ND \\
\hline 50 & $\alpha$-Copaene* & $1,392.73$ & $2.49 \pm 0.42$ & $3.61 \pm 0.67$ & $2.16 \pm 0.21$ & $3.66 \pm 0.97$ \\
\hline 51 & Ketone 2 & $1,397.87$ & $1.84 \pm 0.71$ & $4.41 \pm 1.59$ & ND & ND \\
\hline 52 & Hydrocarbon 5 & $1,400.56$ & $1.43 \pm 0.75$ & ND & $0.92 \pm 0.15$ & $0.68 \pm 0.17$ \\
\hline 53 & Sesquiterpene 1 & $1,403.41$ & $1.03 \pm 0.34$ & $0.69 \pm 0.20$ & $0.44 \pm 0.09$ & $1.14 \pm 0.39$ \\
\hline 54 & Alcohol & $1,415.15$ & $1.25 \pm 0.56$ & $1.40 \pm 0.54$ & $1.27 \pm 0.19$ & $2.62 \pm 0.38$ \\
\hline 55 & Diphenyl oxide & $1,422.94$ & $0.24 \pm 0.05$ & $0.27 \pm 0.11$ & $0.34 \pm 0.05$ & $0.70 \pm 0.34$ \\
\hline 56 & Sesquiterpene 2 & $1,426.15$ & $0.26 \pm 0.06$ & $0.59 \pm 0.23$ & $0.49 \pm 0.07$ & $0.85 \pm 0.29$ \\
\hline 57 & Sesquiterpene 3 & $1,436.26$ & $0.28 \pm 0.09$ & $0.62 \pm 0.22$ & $0.70 \pm 0.06$ & $0.69 \pm 0.16$ \\
\hline 58 & Sesquiterpene 4 & $1,442.48$ & $0.57 \pm 0.22$ & $0.46 \pm 0.17$ & $0.82 \pm 0.11$ & $0.88 \pm 0.54$ \\
\hline 59 & Geranyl acetone* & $1,454.76$ & $9.74 \pm 5.58$ & $5.31 \pm 7.54$ & $3.11 \pm 0.48$ & $9.73 \pm 1.19$ \\
\hline
\end{tabular}

IR=Retention index; ND=Not Detected; *=Confirmed with synthetics; $* *=$ Compared with the NIST library; TMB=Trimethylbenzene.

Table 2. Volatile compounds identified in the treatments applied to soybean pods, cv. FT-Cristalina-RCH (proportions \pm standard error).

\begin{tabular}{llccccc}
\hline No. & \multicolumn{1}{c}{ Compounds } & IR & $\begin{array}{c}\text { Pods with } \\
\text { males }\end{array}$ & $\begin{array}{c}\text { Pods with } \\
\text { females }\end{array}$ & $\begin{array}{c}\text { Pods with } \\
\text { mechanical } \\
\text { damage }\end{array}$ & $\begin{array}{c}\text { Healthy } \\
\text { pods }\end{array}$ \\
\hline $\mathbf{1}$ & a-Pinene* & 919.69 & $0.65 \pm 0.32$ & $\mathrm{ND}$ & $0.47 \pm 0.13$ & $0.50 \pm 0.24$ \\
$\mathbf{2}$ & Pseudocumene (1, 2, 4-TMB)* & 943.04 & $0.29 \pm 0.12$ & $\mathrm{ND}$ & $0.08 \pm 0.03$ & $0.50 \pm 0.17$ \\
$\mathbf{3}$ & Derivate of benzene 1 & 951.6 & $0.54 \pm 0.19$ & $0.58 \pm 0.18$ & $0.42 \pm 0.6$ & $1.19 \pm 0.20$ \\
$\mathbf{4}$ & Derivate of benzene 2 & 955.49 & $0.31 \pm 0.10$ & $0.31 \pm 0.14$ & $0.54 \pm 0.42$ & $0.72 \pm 0.08$ \\
$\mathbf{5}$ & 1-Octen-3-ol* & 970.79 & $3.70 \pm 1.08$ & $3.42 \pm 1.970$ & $18.89 \pm 6.9$ & $\mathrm{ND}$ \\
$\mathbf{6}$ & 6-Methyl-5-hepten-2-one* & 975.46 & $6.46 \pm 2.65$ & $6.52 \pm 2.04$ & $4.29 \pm 2.50$ & $\mathrm{ND}$ \\
$\mathbf{7}$ & 2-Octanone* & 981.56 & $4.91 \pm 0.58$ & $3.52 \pm 1.15$ & $\mathrm{ND}$ & $\mathrm{ND}$ \\
$\mathbf{8}$ & Mesitylene* & 988.17 & $2.52 \pm 0.54$ & $2.16 \pm 0.41$ & $\mathrm{ND}$ & $3.22 \pm 0.35$ \\
$\mathbf{9}$ & (Z)-3-Hexenyl acetate* & 995.76 & $1.10 \pm 0.18$ & $0.94 \pm 0.11$ & $18.58 \pm 6.49$ & $1.08 \pm 0.17$ \\
$\mathbf{1 0}$ & Terpene 1 & $1,003.71$ & $0.98 \pm 0.09$ & $0.31 \pm 0.11$ & $1.85 \pm 0.75$ & $0.61 \pm 0.09$ \\
\hline
\end{tabular}

$\mathrm{IR}=$ Retention index; ND=Not detected; $*=$ Confirmed with synthetics; $* *=$ Compared with the NIST library; TMB=Trimethylbenzene. 
Table 2. Continued...

\begin{tabular}{|c|c|c|c|c|c|c|}
\hline No. & Compounds & IR & $\begin{array}{l}\text { Pods with } \\
\text { males }\end{array}$ & $\begin{array}{l}\text { Pods with } \\
\text { females }\end{array}$ & $\begin{array}{c}\text { Pods with } \\
\text { mechanical } \\
\text { damage }\end{array}$ & $\begin{array}{l}\text { Healthy } \\
\text { pods }\end{array}$ \\
\hline 11 & Cymene isomer 1 & $1,014.7$ & $0.51 \pm 0.15$ & $0.98 \pm 0.16$ & $0.30 \pm 0.05$ & $1.38 \pm 0.17$ \\
\hline 12 & Hemellitol $(1,2,3-\mathrm{TMB})^{*}$ & $1,018.95$ & $2.16 \pm 0.16$ & $0.48 \pm 0.17$ & $0.22 \pm 0.06$ & $1.18 \pm 0.24$ \\
\hline 13 & Ethyl hexanol* & $1,023.19$ & $1.70 \pm 0.24$ & $4.38 \pm 1.43$ & $1.08 \pm 0.22$ & $4.67 \pm 1.59$ \\
\hline 14 & $(Z)-\beta$-Ocimene* & $1,026.17$ & $0.08 \pm 0.06$ & $0.97 \pm 0.49$ & $0.78 \pm 0.45$ & $2.27 \pm 0.50$ \\
\hline 15 & Limonene* & $1,029.14$ & $0.24 \pm 0.11$ & ND & $0.15 \pm 0.07$ & $0.72 \pm 0.36$ \\
\hline 16 & Unknown 1 & $1,036.36$ & $0.25 \pm 0.14$ & $0.02 \pm 0.02$ & $0.08 \pm 0.04$ & $0.12 \pm 0.08$ \\
\hline 17 & $(E)-\beta$-Ocimene* & $1,039.76$ & $0.27 \pm 0.15$ & ND & $2.34 \pm 0.61$ & ND \\
\hline 18 & Salicylaldehyde** & $1,049.32$ & $0.33 \pm 0.12$ & $0.23 \pm 0.11$ & $0.23 \pm 0.09$ & $0.54 \pm 0.09$ \\
\hline 19 & Cymene isomer 2 & $1,055.69$ & $0.59 \pm 0.37$ & $0.11 \pm 0.11$ & $0.06 \pm 0.06$ & $0.32 \pm 0.17$ \\
\hline 20 & Unknown 2 & $1,066.95$ & ND & ND & ND & ND \\
\hline 21 & Cymene isomer 3 & $1,076.51$ & $0.60 \pm 0.14$ & $0.14 \pm 0.09$ & $0.25 \pm 0.05$ & $0.85 \pm 0.16$ \\
\hline 22 & Cymene isomer 4 & $1,079.48$ & $5.86 \pm 0.60$ & $0.11 \pm 0.07$ & $0.41 \pm 0.08$ & $0.64 \pm 0.12$ \\
\hline 23 & Alkene 1 & $1,086.49$ & $0.44 \pm 0.17$ & ND & ND & ND \\
\hline 24 & Hydrocarbon 1 & $1,094.7$ & $1.97 \pm 0.11$ & ND & $0.99 \pm 0.17$ & $0.67 \pm 0.24$ \\
\hline 25 & Linalool* & $1,097.54$ & $1.33 \pm 0.27$ & ND & $0.71 \pm 0.08$ & ND \\
\hline 26 & 2-Nonen-1-ol & $1,102.84$ & $0.52 \pm 0.12$ & $6.53 \pm 1.77$ & $5.25 \pm 1.31$ & $3.71 \pm 0.45$ \\
\hline 27 & Unknown 3 & $1,128.99$ & ND & ND & ND & ND \\
\hline 28 & Carveol & $1,158.12$ & ND & $0.34 \pm 0.16$ & $0.41 \pm 0.05$ & $0.61 \pm 0.13$ \\
\hline 29 & Aldehyde 1 & $1,171.19$ & $6.34 \pm 1.46$ & $0.96 \pm 0.44$ & $5.01 \pm 3.36$ & $1.05 \pm 0.24$ \\
\hline 30 & Ketone 1 & $1,192.29$ & $1.29 \pm 0.57$ & $1.47 \pm 0.37$ & ND & ND \\
\hline 31 & Hydrocarbon 2 & $1,196.88$ & $2.11 \pm 1.38$ & $0.71 \pm 0.19$ & $0.70 \pm 0.08$ & $1.11 \pm 0.19$ \\
\hline 32 & Unknown 4 & $1,201.11$ & $0.92 \pm 0.26$ & $0.12 \pm 0.12$ & ND & ND \\
\hline 33 & Methyl salicylate* & $1,204.43$ & ND & ND & ND & ND \\
\hline 34 & Aldehyde 2 & $1,207.65$ & $0.56 \pm 0.12$ & $11.42 \pm 2.74$ & $9.09 \pm 0.89$ & $11.88 \pm 1.44$ \\
\hline 35 & Derivate of cyclohexanone & $1,269.18$ & $1.04 \pm 0.38$ & $0.96 \pm 0.32$ & $0.22 \pm 0.08$ & ND \\
\hline 36 & Geranial* & $1,276.06$ & $0.76 \pm 0.16$ & $0.53 \pm 0.20$ & $0.54 \pm 0.11$ & $0.93 \pm 0.36$ \\
\hline 37 & Ester 1 & $1,280.89$ & ND & ND & ND & ND \\
\hline 38 & Unknown 5 & $1,281.84$ & $0.33 \pm 0.13$ & $0.65 \pm 0.23$ & $0.50 \pm 0.08$ & $0.82 \pm 0.0 .18$ \\
\hline 39 & 2-Undecanone* & $1,295.17$ & $4.42 \pm 0.93$ & $0.57 \pm 0.19$ & ND & ND \\
\hline 40 & Hydrocarbon 3 & $1,299.09$ & ND & ND & ND & ND \\
\hline 41 & Unknown 6 & $1,298.94$ & $0.37 \pm 0.24$ & $0.97 \pm 0.16$ & $0.42 \pm 0.07$ & $1.55 \pm 0.40$ \\
\hline 42 & Hydrocarbon 4 & $1,307.14$ & $2.29 \pm 0.96$ & $1.42 \pm 0.24$ & $0.23 \pm 0.11$ & $2.07 \pm 0.35$ \\
\hline 43 & Aldehyde 3 & $1,311.93$ & $14.05 \pm 4.11$ & $0.61 \pm 0.14$ & $0.57 \pm 0.12$ & $0.76 \pm 0.13$ \\
\hline 44 & Unknown 7 & $1,315.23$ & ND & ND & ND & ND \\
\hline 45 & Ester 2 & $1,325.45$ & ND & ND & ND & ND \\
\hline 46 & Ester 3 & $1,361.18$ & $8.25 \pm 2.42$ & $4.71 \pm 0.93$ & $5.71 \pm 2.25$ & $3.42 \pm 1.35$ \\
\hline 47 & Unknown 8 & $1,377.42$ & $2.22 \pm 2.22$ & $0.14 \pm 0.14$ & $0.28 \pm 0.12$ & ND \\
\hline 48 & Ester 4 & $1,382.48$ & $7.01 \pm 4.22$ & $3.17 \pm 1.05$ & $4.23 \pm 1.64$ & $6.84 \pm 1.17$ \\
\hline 49 & (Z)-8-Dodecenyl acetate (Ester 5) & $1,385.94$ & $1.44 \pm 0.35$ & $17.00 \pm 6.36$ & ND & ND \\
\hline 50 & $\alpha$-Copaene* & $1,392.07$ & ND & $10.73 \pm 2.99$ & $8.20 \pm 1.96$ & $12.40 \pm 2.99$ \\
\hline 51 & Ketone 2 & $1,397.87$ & ND & ND & ND & ND \\
\hline 52 & Hydrocarbon 5 & $1,399.52$ & $1.23 \pm 0.52$ & $0.02 \pm 0.02$ & $1.01 \pm 0.29$ & $0.91 \pm 0.38$ \\
\hline 53 & Sesquiterpene 1 & $1,403.15$ & $2.75 \pm 0.69$ & $1.45 \pm 0.22$ & ND & $11.97 \pm 8.67$ \\
\hline 54 & Alcohol & $1,414.41$ & $2.64 \pm 0.66$ & $1.68 \pm 0.33$ & $1.35 \pm 0.31$ & $3.15 \pm 0.98$ \\
\hline 55 & Diphenyl oxide & $1,422.3$ & $0.38 \pm 0.22$ & ND & $0.29 \pm 0.10$ & ND \\
\hline 56 & Sesquiterpene 2 & $1,426.24$ & $1.26 \pm 0.53$ & $1.60 \pm 0.29$ & $0.18 \pm 0.06$ & $2.16 \pm 0.57$ \\
\hline 57 & Sesquiterpene 3 & $1,435.25$ & ND & $2.34 \pm 0.60$ & $0.70 \pm 0.11$ & $3.26 \pm 0.75$ \\
\hline 58 & Sesquiterpene 4 & $1,441.16$ & ND & $4.71 \pm 0.67$ & $0.37 \pm 0.11$ & $5.66 \pm 0.74$ \\
\hline 59 & Geranyl acetone* & $1,453.83$ & ND & $0.66 \pm 0.66$ & $2.04 \pm 0.26$ & $4.58 \pm 3.61$ \\
\hline
\end{tabular}

$\mathrm{IR}=$ Retention index; ND=Not detected; *=Confirmed with synthetics; $* *=$ Compared with the NIST library; TMB=Trimethylbenzene. 
<smiles>CC1=CCC2CC1C2(C)C</smiles>

$\alpha$-Pinene<smiles>CCCCCCC(C)=O</smiles>

2-Octanone<smiles>CCCCC(CC)CO</smiles>

Ethyl hexanol<smiles>Cc1ccc(C)c(C)c1</smiles>

Pseudocumene (1, 2, 4-TMB)<smiles>CC1CC(C)CC(C)C1</smiles>

Mesitylene<smiles>C=C/C(C)=C\CC=C(C)C</smiles>

(Z)- $\beta$-Ocimene<smiles>C=CC(C)(O)CCC=C(C)C</smiles>

Linalool<smiles>C=CC(O)CCCCC</smiles>

1-Octen-3-ol<smiles>CC/C=C\CCOC(C)=O</smiles>

(Z)-3-Hexenyl acetate<smiles>C=C(C)C1CC=C(C)CC1</smiles>

Limonene<smiles>COC(=O)c1ccccc1O</smiles>

Methyl salicylate<smiles>CC1CC[C@H](C(C)C)[C@@H]2C(C)CC[C@@H]12</smiles>

$\alpha$-Copaene<smiles>CC(=O)CCC=C(C)C</smiles>

6-Methyl-5-hepten-2-one<smiles>Cc1cccc(C)c1C</smiles>

Hemellitol (1, 2, 3-TMB)<smiles>C=CC(C)=CCC=C(C)C</smiles>

(E)- $\beta$-Ocimeno<smiles>CC(C)=CCC/C(C)=C/C=O</smiles>

Geranial

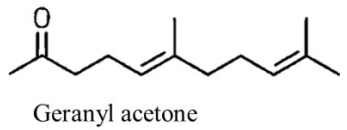

Geranyl acetone

2-Undecanone

Figure 1. Structures of identified compounds from soybean plants. TMB $=$ trimethylbenzene.

The compounds mostly produced by soybean plant infested by $R$. nigerrimus males and females were 1-octen-3-ol, 6-metil-5-hepten-2-one, $(E)$ - $\beta$-ocimene, salicylaldehyde, alkene 1, linalool, methyl salicylate, (Z)-8-dodecenyl acetate (ester 5), ketone 2 and geranyl acetone (Figure 2). The main compounds were 1-octen-3-ol and (Z)-8-dodecenyl acetate (ester 5 ), produced during infestation by males, and $(E)$ - $\beta$-ocimene and (Z)-8-dodecenyl acetate (ester 5), produced during infestation by females (Table 1 ). Figure 3 shows that there are significant differences (OOB-rate of estimated error $=0 \%$ ) in the areas of peaks between healthy, mechanically damaged and infested plants. Table 3 indicates plant grouping by identified compounds.

The compounds mostly produced by soybean pods infested by male and female $R$. nigerrimus were (Z)-8-dodecenyl acetate, aldehyde 2 y 3 , $\alpha$-copaene, ester 3 y 4 , 6-methyl-5hepten-2-one, 2-nonen-1-ol, cymene isomer 4 and aldehyde 1 (Figure 4). The main compounds were aldehyde 3 and ester 3 and 4 produced during infestation by males, and (Z)-8-dodecenyl acetate (ester 5), aldehyde 2 and $\alpha$-copaene, produced during infestation by females (Table 2). Figure 5 shows that there are significant differences (OOB-estimated error rate $=8.33 \%$ ) in the areas of peaks between healthy, mechanically damaged and infested plants. Table 4 indicates plant groupings by identified compounds.

\section{Discussion}

This study revealed that the volatile profile of healthy soybean plants differs from the volatile profiles of plants damaged mechanically and by $R$. nigerrimus, both qualitatively and quantitatively. Of the 19 identified compounds, 12 had not been reported in previous studies on volatiles emitted by soybean (Liu et al., 1989; Boué et al., 2003; Zhu and Park, 2005; Cai et al., 2015; Cortés, 2016).

Regarding quantitative differences, the results of this study demonstrated that four of the compounds produced by plants infested by females ( $(E) \beta$-ocimene, linalool, methyl salicylate and $\alpha$-copaene) were produced in greater proportion than by plants in the other treatments and the control. Recent 


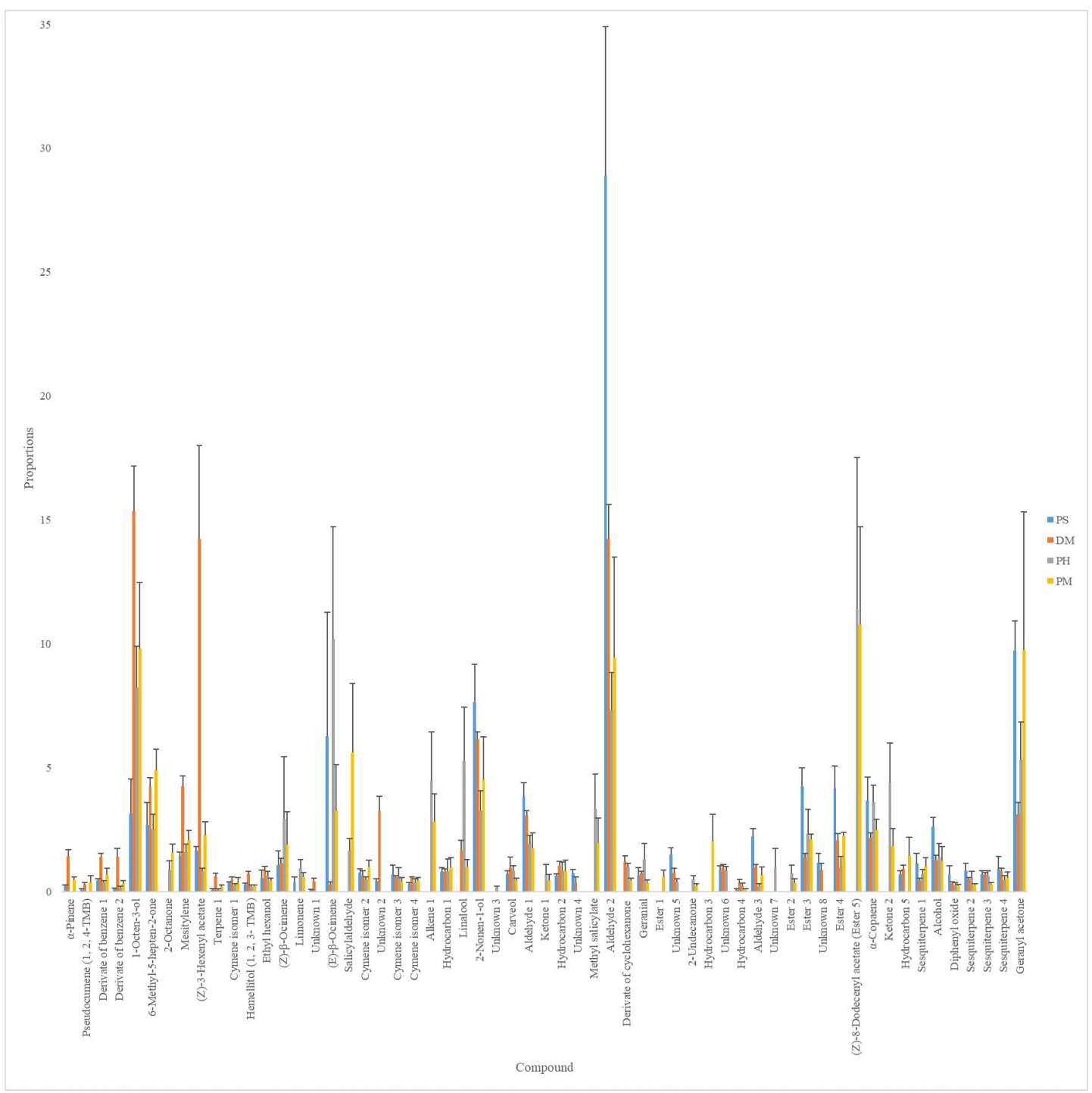

Figure 2. Qualitative and quantitative comparison of the volatiles from soybean plant.

Table 3. Classification matrix of soybean plants (class. error).

\begin{tabular}{ccccc}
\hline & DM & PH & PM & PS \\
\hline DM & 6 & 0 & 0 & 0 \\
PH & 0 & 6 & 0 & 0 \\
PM & 0 & 0 & 6 & 0 \\
PS & 0 & 0 & 0 & 6 \\
\hline
\end{tabular}

Table 4. Classification matrix of soybean pods (Class.Error)

\begin{tabular}{ccccc}
\hline & PSp & DMp & PHp & PMp \\
\hline PSp & 5 & 0 & 1 & 0 \\
DMp & 0 & 6 & 0 & 0 \\
PHp & 1 & 0 & 5 & 0 \\
PMp & 0 & 0 & 0 & 6 \\
\hline
\end{tabular}

$\mathrm{PSp}=$ healthy pods; $\mathrm{DMp}=$ mechanically damaged pods;

$\mathrm{PHp}=$ pods damaged by females; $\mathrm{PMp}=$ pods damaged by males.

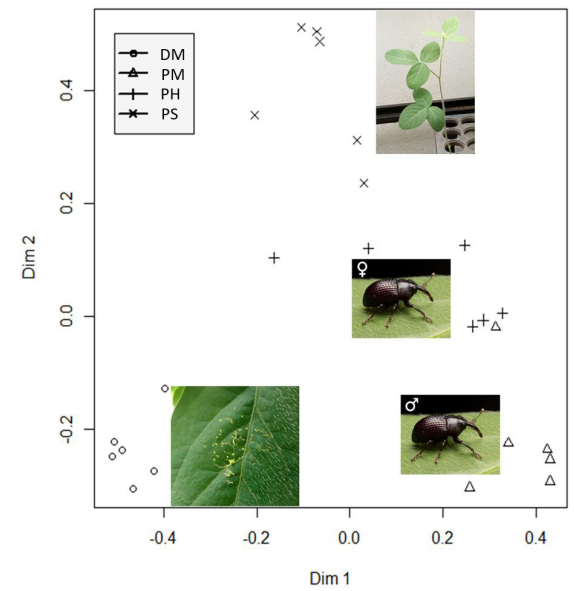

Figure 3. Relationship of compounds identified from plant mechanically damaged (DM), by females $(\mathrm{PH})$ and by males (PM) in contrast with healthy plant (PS). 


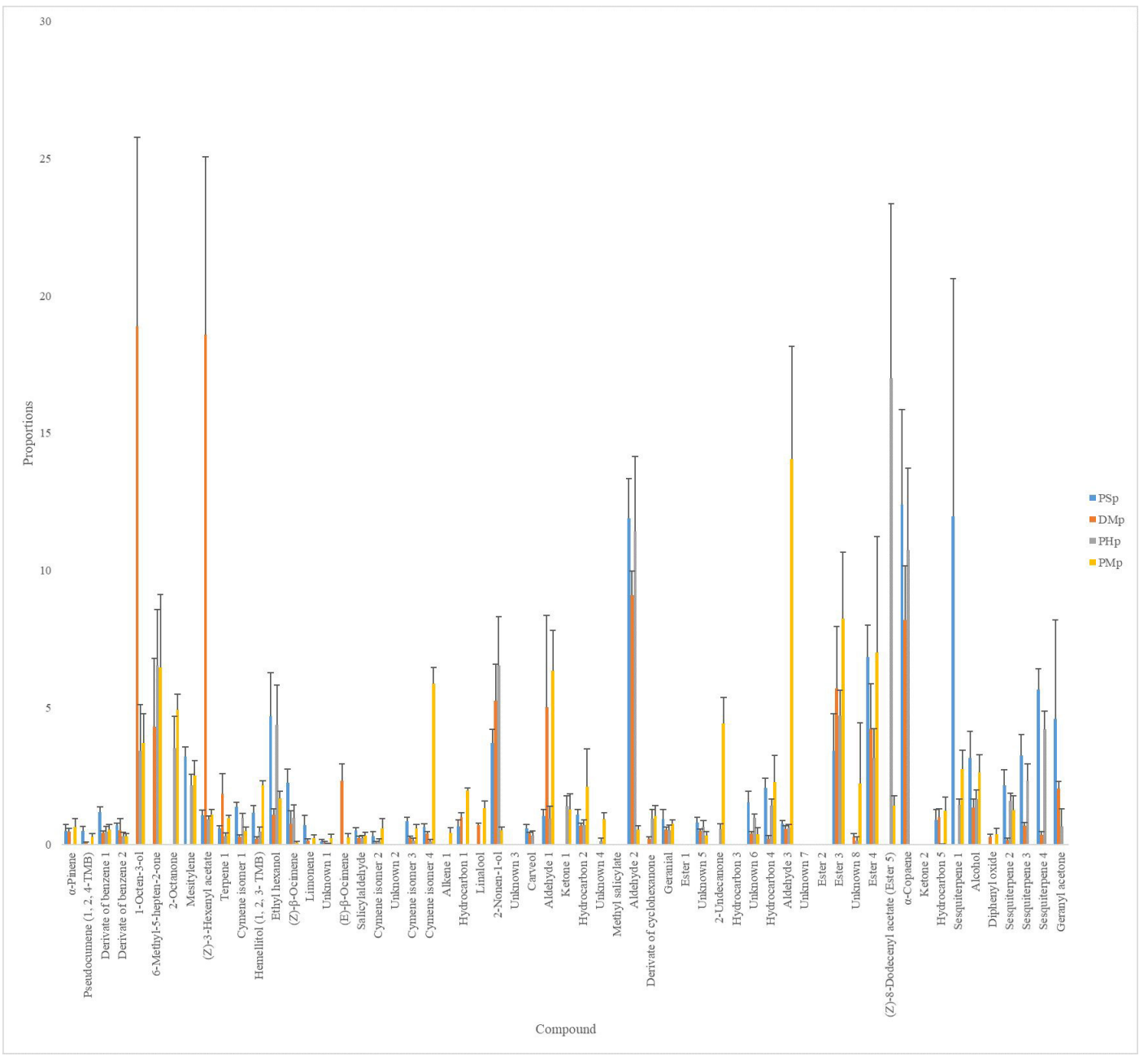

Figure 4. Qualitative and quantitative comparison of the volatiles from soybean pods.

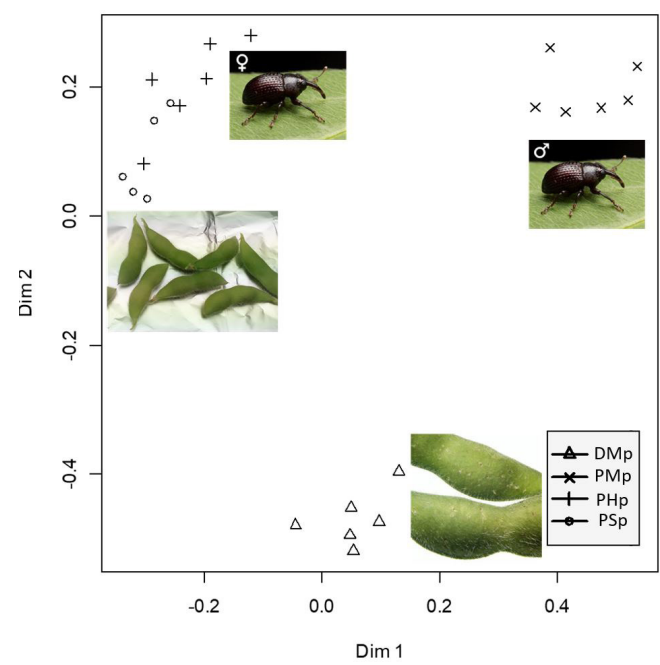

Figure 5. Relationship among compounds identified from soybean pods damaged mechanically (DMp), by females (PMp) and by males (PHp) in contrast with healthy pods (Psp). studies report that the reason that volatiles such as linalool and methyl salicylate in plants increase is because they are HIPVs produced in response to herbivory (War et al., 2011; García, 2017). Emission of these compounds has also been detected in Capsicum spp. and Camellia sinensis, host plants of chili and tea weevils, respectively. Studies on these insect-plant interactions have demonstrated that induced volatiles, such as benzylic alcohol, (Z)-3-hexenal, myrcene, benzaldehyde and $\gamma$-terpinene, attract conspecific weevils of both sexes, while linalool and (Z)-3-hexenyl acetate attracts only females, and $(E)$ - $\beta$-ocimene attracts only males (Muñiz-Merino et al., 2014; Sun et al., 2010). This suggests that the soybean weevil can respond similarly to these volatiles. It has been reported that $(E)$ - $\beta$-ocimene is a volatile commonly released by leaves and flowers of diverse plant species, inducing plant defense response and establishing tritrophic interactions as well as promoting mating and oviposition of insect pests (Dicke and Baldwin, 2010; Farré-Armengol et al., 2017; Tang et al., 2016).

Under the condition of infestation by females and males of the black soybean weevil, salicylaldehyde and 
2-octanone were detected. These compounds had not been previously reported in soybean plants (Liu et al., 1989). Possibly, salicylaldehyde was detected because it is a precursor of methyl salicylate (Asghari \& Mosaeybi, 2009), which was also detected in the insect-plant treatments. Also, 2-octanone is a volatile compound that is not produced by plants; it is possibly produced by the weevils while they feed as a signal for other insect species and/or their conspecifics (Meiners et al., 2003). The presence of methyl salicylate and (Z)-3-hexenyl acetate has been reported in the soybean varieties London and Davis (Boué et al., 2003; Moraes et al., 2008). Dong et al. (2011) report that Camellia sinensis plants emit numerous volatile compounds, such as (Z)-3-hexen-1-ol, linalool, $\alpha$-farnesene, benzylic nitryl, indol, nerolidol, and ocimene, in high concentrations as a defense response to herbivore attack. They also underline that, for identification of induced volatile compounds, not only is it necessary to consider qualitative differences but also quantitative differences compared with the control without damage since, depending on the concentration in which these metabolites are found, they will cause an effect on the behavior of conspecifics and of parasitoids.

The study conducted by Moraes et al. (2008) indicates that (Z)-3-hexenyl acetate is produced when vegetative tissue is damaged by herbivory. Some compounds produced by soybeans are considered components of plant defense against herbivores are (Z)-3-hexenyl acetate, farnesene, caryophyllene and humulene (Cai et al., 2015). In our study, we observed that the soybean plant (cv FT-Cristalina- $\mathrm{RCH}$ ) emitted higher concentrations of (Z)-3-hexenyl acetate when damaged mechanically than when damaged by insects (Figure 2). This difference in emission of volatiles depends on both the physiological state of the soybean plant and the variety of soybean when it is attacked by an insect pest (Liu et al., 1989; Rostás and Eggert, 2008).

However, this study should be complemented with additional experiments to determine the total compounds present in this variety of soybean as well as their effect on the insect pest and on natural enemies.

\section{Conclusion}

Our study demonstrates that the soybean plant (cv FT-Cristalina- $\mathrm{RCH}$ ) produces induced volatiles in response to damage, both mechanical and that produced by $R$. nigerrimus. Of the compounds found, 19 represented differences between healthy plants and those infested by females or males or those mechanically damaged. These compounds include 1-octen-3-ol, 6-methyl-5-hepten-2-one, 2-octanone, $(Z)$ - $\beta$-ocimene, limonene, $(E)$ - $\beta$-ocimene, salicylaldehyde, linalool and methyl salicylate.

\section{Acknowledgements}

We thank the soybean growers who allowed us to collect soybean weevils and funding through the CONACYT INFR-2014-01(224846) and SEP-CONACYT (CB20172018; A1-S-23359) projects.

\section{References}

ASGHARI, G. and MOSAEYBI, M., 2009. Biotransformation of salicylaldehyde to salicin using Varthemia persica cell suspension cultures. Indian Journal of Pharmaceutical Sciences, vol. 5, no. 2, pp. 109-114.

BOUÉ, S.M., SHIH, B.Y., CARTER-WIENTJES, C.H. and CLEVELAND, T.E., 2003. Identification of volatile compounds in soybean at various developmental stages using solid phase microextraction. Journal of Agricultural and Food Chemistry, vol. 51, no. 17, pp. 4873-4876. http://dx.doi.org/10.1021/jf030051q. PMid:12903938.

BYERS, K.J., BRADSHAW JUNIOR, H.D. and RIFFELL, J.A., 2014. Three floral volatiles contribute to differential pollinator attraction in monkeyflowers (Mimulus). The Journal of Experimental Biology, vol. 217, no. Pt 4, pp. 614-623. http:// dx.doi.org/10.1242/jeb.092213. PMid:24198269.

CAI, L., KOZIEL, J.A. and O’NEAL, M.E., 2015. Studying plant-insect interactions with solid phase microextraction: screening for airborne volatile emissions response of soybeans to the soybean aphid, Aphis glycines Matsumura (Hemiptera: Aphididae). Chromatography (Basel), vol. 2, no. 2, pp. 265-276. http://dx.doi.org/10.3390/chromatography2020265.

CORTÉS, F., 2016. Atracción del picudo de la soya (Rhyssomatus nigerrimus) a los volátiles de la soya. México: Instituto Tecnológico de Tapachula, 35 p. Tesis de licenciatura.

DICKE, M. and BALDWIN, I.T., 2010. The evolutionary context for herbivore-induced plant volatiles: beyond the 'cry for help'. Trends in Plant Science, vol. 15, no. 3, pp. 167-175. http://dx.doi. org/10.1016/j.tplants.2009.12.002. PMid:20047849.

DONG, F., YANG, Z., BALDERMANN, S., SATO, Y., ASAI, T. and WATANABE, N., 2011. Herbivore-induced volatiles from tea (Camellia sinensis) plants and their involvement in intraplant communication and changes in endogenous nonvolatile metabolites. Journal of Agricultural and Food Chemistry, vol. 59, no. 24, pp. 13131-13135. http://dx.doi.org/10.1021/jf203396a. PMid:22077631

FARRÉ-ARMENGOL, G., FILELLA, I., LLUSIÀ, J. and PEÑUELAS, J., 2017. $\beta$-Ocimene, a key floral and foliar volatile involved in multiple interactions between plants and other organisms. Molecules (Basel, Switzerland), vol. 22, no. 7, pp. 1148. http:// dx.doi.org/10.3390/molecules22071148. PMid:28703755.

FOOD AND AGRICULTURE ORGANIZATION - FAO, 2017 [viewed 16 November 2017]. Oilseeds and oilseed products [online]. Available from: http://www.fao.org/3/a-BT087e.pdf

GARCÍA, G. (2017). El papel de la olfacción en la selección del hospedante por Trichoplusia ni (Hübner). México: El Colegio de la Frontera Sur, 47 p. Tesis Doctorado en Ciencias en Ecología y Desarrollo Sustentable.

LIAW, A. and WIENER, M., 2002. Classification and regression by randomForest. $R$ News, vol. 2-3, pp. 18-22.

LIU, S.H., NORRIS, D.M. and LYNE, P., 1989. Volatiles from the foliage of soybean, Glycine max, and lima bean, Phaseolus lunatus: their behavioral effects on the insects Trichoplusia ni and Epilachna varivestis. Journal of Agricultural and Food Chemistry, vol. 37, no. 2, pp. 496-501. http://dx.doi.org/10.1021/jf00086a050.

LÓPEZ-GUILLÉN, G., VALDEZ-CARRASCO, J., GÓMEZ RUIZ, J., MARTÍNEZ ZARATE, C.J. and CRUZ-LÓPEZ, L., 2016. Sexual dimorphism and ratio of natural populations of 
Rhyssomatus nigerrimus adults. Southwestern Entomologist, vol. 41, pp. 837-844. http://dx.doi.org/10.3958/059.041.0325.

MARÍN, J. and CÉSPEDES, C., 2007 [viewed 16 November 2017]. Compuestos volátiles de plantas, origen, emisión, efectos, análisis y aplicaciones al agro. Revista Fitotecnia Mexicana [online], vol. 30, no. 4, pp. 327-351. Available from: http://www.redalyc.org/ articulo.oa? $\mathrm{id}=61030401$

MEINERS, T., WÄCKERS, F. and LEWIS, W.J., 2003. Associative learning of complex odours in parasitoid host location. Chemical Senses, vol. 28, no. 3, pp. 231-236. http://dx.doi.org/10.1093/ chemse/28.3.231. PMid:12714445.

MICHEREFF, M.F.F., LAUMANN, R.A., BORGES, M., MICHEREFF-FILHO, M., DINIZ, I.R., FARIAS NETO, A.L. and MORAES, M.C.B., 2011. Volatiles mediating a plant-herbivorenatural enemy interaction in resistant and susceptible soybean cultivars. Journal of Chemical Ecology, vol. 37, no. 3, pp. 273-285. http://dx.doi.org/10.1007/s10886-011-9917-4. PMid:21318397.

MORAES, C.M., LEWIS, W.J. and TUMLINSON, J.H., 2000. Examining plant-parasitoid interactions in tritrophic systems. Anais da Sociedade Entomológica do Brasil, vol. 29, no. 2, pp. 189-203. http://dx.doi.org/10.1590/S0301-80592000000200001.

MORAES, M.C., PAREJA, M., LAUMANN, R.A., HOFFMANNCAMPO, C.B. and BORGES, M., 2008. Response of the parasitoid Telenomus podisi to induced volatiles from soybean damaged by stink bug herbivory and oviposition. Journal of Plant Interactions, vol. 3, no. 2, pp. 111-118. http://dx.doi. org/10.1080/17429140701810724.

MORAES, M.C.B., LAUMANN, R., SUIJI, E.R., PIRES, C. and BORGES, M., 2005. Induced volatiles in soybean and pigeon pea plants artificially infested with the neotropical brown stink bug, Euschitus heros, and their effect on the egg parasitoid, Telenomus podisi. Entomologia Experimentalis et Applicata, vol. 115, no. 1, pp. 227-237. http://dx.doi.org/10.1111/j.1570-7458.2005.00290.x.

MUÑIZ-MERINO, M., CIBRIÁN-TOVAR, J., HIDALGO-MORENO, C., BAUTISTA-MARTÍNEZ, N., VAQUERA-HUERTA, H. and ALDAMA-AGUILERA, C., 2014. Compuestos volátiles atraen al picudo (Anthonomus eugenii Cano) del chile (Capsicum spp.) y presentan sinergia con su feromona de agregación. Agrociencia, vol. 48 , no. 8 , pp. $819-832$.

ROSTÁS, M. and EGGERT, K., 2008. Ontogenetic and spatiotemporal patterns of induced volatiles in Glycine max in the light of the optimal defence hypothesis. Chemoecology, vol. 18, no. 1, pp. 29-38. http://dx.doi.org/10.1007/s00049-007-0390-z.
SÁNCHEZ, V. and DÉLANO, J., 2003. La emisión de compuestos volátiles en plantas ¿Sutil señal reconocida tanto por plantas como por insectos? Folia Entomologica Mexicana, vol. 42, no. 2, pp. 273-290.

SERVICIO DE INFORMACIÓN AGROALIMENTARIA Y PESQUERA - SIAP, 2017 [viewed 16 November 2017]. Resumen Nacional Intención de siembra 2018 [online]. Available from: http://infosiap.siap.gob.mx/opt/agricultura/intension/ Intenci $\% \mathrm{C} 3 \% \mathrm{~B} 3 \mathrm{n} \% 20 \mathrm{de} \% 20$ siembraOI\%202018porcultivo.pdf

SUN, X.L., WANG, G.C., CAI, X.M., JIN, S., GAO, Y. and CHEN, Z.M., 2010. The tea weevil, Myllocerinus aurolineatus, is attracted to volatiles induced by conspecifics. Journal of Chemical Ecology, vol. 36, no. 4, pp. 388-395. http://dx.doi.org/10.1007/ s10886-010-9771-9. PMid:20349338.

TANG, R., ZHANG, F. and ZHANG, Z.N., 2016. Electrophysiological Responses and Reproductive Behavior of Fall Webworm Moths (Hyphantria cunea Drury) are Influenced by Volatile Compounds from Its Mulberry Host (Morus alba L.). Insects, vol. 7, no. 2, pp. 19. http://dx.doi.org/10.3390/insects7020019. PMid:27153095.

TERÁN VARGAS, A.P. and LÓPEZ GUILLÉN, G., 2014 [viewed 16 November 2017] El picudo de la soya Rhyssomatus nigerrimus Fahraeus 1837 (Coleóptera: Curculionidae). Instituto Nacional de Investigaciones Forestales [online]. Villa Cuauhtémoc, Tam: Centro de Investigación Regional del Noreste Campo Experimental Las Huastecas. Folleto Técnico, no. MX-0-310304-47-03-14-0938. Available from: http://www.inifapcirne.gob.mx/Biblioteca/ Publicaciones/1003.pdf

VISSER, J.H., 1986. Host odor perception in phytophagous insects. Annual Review of Entomology, vol. 31, no. 1, pp. 121-144. http:// dx.doi.org/10.1146/annurev.en.31.010186.001005.

VIVANCO, J., COSIO, E., LOYOLA, V. and FLORES, H., 2005. Mecanismos químicos de defensa en las plantas. Investigacion y Ciencia, vol. 341, pp. 68-75.

WAR, A.R., SHARMA, H., PAULRAJ, M., WAR, M. and IGNACIMUTHU, S., 2011. Herbivore induced plant volatiles their role in plant defense for pest management. Plant Signaling \& Behavior, vol. 6, no. 12, pp. 1973-1978. http://dx.doi.org/10.4161/ psb.6.12.18053. PMid:22105032.

ZHU, J. and PARK, K.C., 2005. Methyl salicylate, a soybean aphid-induced plant volatile attractive to the predator Coccinella septempunctata. Journal of Chemical Ecology, vol. 31, no. 8, pp. 1733-1746. http://dx.doi.org/10.1007/s10886-005-5923-8. PMid:16222805. 